\title{
COVID-19 Vaccine Hesitancy among Health Care Workers
}

\author{
Seung-Won $\mathrm{Oh}^{*}$ \\ Department of Family Medicine, Seoul National University Hospital Healthcare System Gangnam Center, Seoul, Korea
}

\section{See original paper on 445}

As of November 5, 2021, more than 248 million severe acute respiratory syndrome coronavirus 2 (SARS-CoV-2) infections and 5 million coronavirus disease 2019 (COVID-19) deaths have been reported. ${ }^{1)}$ The 73rd World Health Assembly passed a resolution in May 2020 acknowledging the need for widespread immunization as a worldwide public health measure for preventing, containing, and terminating SARS-CoV-2 transmission. ${ }^{2)}$ There are now over 155 candidate vaccines worldwide and 485 trials in progress, and 23 COVID-19 vaccines have been approved by at least one country. ${ }^{3)}$ Since BioNTech and Pfizer's BNT162 vaccine first received emergency use authorization in December 2020, 3.9 billion people have received at least one dose. ${ }^{1)}$ However, many countries are experiencing a slowdown in vaccination rates. In the United States, the full vaccination rate has not exceeded $60 \%$ since reaching $50 \%$ in July 2021 . Since then, the delta mutation has rapidly increased the number of infected people. This slowdown in vaccination rates is mainly attributed to vaccine hesitancy.

Delayed acceptance or refusal of the vaccination despite its availability, known as "vaccine hesitancy," was already a growing concern before the COVID-19 pandemic. ${ }^{4)}$ Vaccine hesitancy is influenced by a variety of factors, including knowledge and prior experience. It is also the outcome of broader effects, and it should always be considered in the context of the historical, political, and socio-cultural contexts in which vaccinations occur. The impact of these factors on vaccine hesitancy is also mediated by trust in the vaccine delivery system, health care professionals who suggest and administer vaccines, policymakers who determine vaccination programs, and the various forms of vaccine information transmitted in the media. ${ }^{5)}$
Understanding whether people are willing to be vaccinated, the reasons for their willingness or unwillingness, and the most trusted sources of information in their decision-making are required to promote vaccine acceptance.

In the present issue, Kumar et al. ${ }^{6}$ investigated the willingness to receive the COVID-19 vaccination and associated factors among 599 Indian health care workers (HCWs) from February to March 2021. This survey found that $73 \%$ of HCWs were willing to accept the vaccines, while $10.9 \%$ refused and $16.2 \%$ needed more time to decide. ${ }^{6)}$ A survey of HCWs, who should have a scientific attitude about vaccines, reported a surprising $27 \%$ vaccine hesitancy rate. However, studies conducted in other countries have reported similar results.

According to a survey conducted in Israel in March 2020, $78 \%$ of physicians and $61 \%$ of nurses intended to receive a COVID-19 vaccine. ${ }^{7)}$ As of April 2020, an estimated 63\% of Hong Kong nurses intended to take the COVID-19 vaccine. ${ }^{8)}$ In late 2020 , only $36 \%$ of United States HCWs said they were willing to take the vaccine as soon as it became available.9 ${ }^{9}$ On the other hand, according to a survey conducted between March and May 2021, after vaccinations began in the United States, $85 \%$ of HCWs answered that they had been or would be vaccinated. ${ }^{10)}$

HCWs bridge the gap between health care policymakers and patients and influence patients' vaccine decisions. Vaccination rates among HCWs correlate positively with their willingness to recommend COVID-19 vaccination to their patients. ${ }^{9}$ Therefore, identifying the factors influencing vaccine hesitancy in HCW has important implications for increasing the inoculation rate of the entire population. In the study of Kumar et al., ${ }^{6}$ individuals who considered themselves at risk of disease were more likely to accept the COVID-19 vaccination. Additionally, the most important reason for vaccine hesi- 
tancy was safety and efficacy concerns. ${ }^{6}$ This is also a common result of previous studies. Accordingly, more active dissemination of scientific evidence about COVID-19 transmission patterns and vaccine safety will contribute to reducing vaccine hesitancy and increasing vaccination rates. Additional studies should also be conducted to identify factors affecting vaccine hesitancy in various groups.

\section{CONFLICT OF INTEREST}

No potential conflict of interest relevant to this article was reported.

\section{ORCID}

Seung-Won Oh: https://orcid.org/0000-0003-3800-0754

\section{REFERENCES}

1. World Health Organization. WHO Coronavirus (COVID-19) Dashboard [Internet]. Geneva: World Health Organization; 2021 [cited 2021 Nov 5]. Available from: https://covid19.who.int/.

2. World Health Organization. COVID-19 response [Internet]. Geneva: World Health Organization; 2020 [cited 2021 Nov 5]. Available from: https://apps.who.int/gb/ebwha/pdf_files/WHA73/A73_R1-en.pdf.
3. McGill COVID19 Vaccine Tracker Team. Vaccines candidates in clinical trials [Internet]. Montreal: McGill University, McGill COVID19 Vaccine Tracker Team; 2021 [cited 2021 Nov 5]. Available from: https:// covid19.trackvaccines.org/vaccines/.

4. Dube E, Laberge C, Guay M, Bramadat P, Roy R, Bettinger J. Vaccine hesitancy: an overview. Hum Vaccin Immunother 2013;9:1763-73.

5. Larson HJ, Cooper LZ, Eskola J, Katz SL, Ratzan S. Addressing the vaccine confidence gap. Lancet 2011;378:526-35.

6. Kumar R, Beniwal K, Bahurupi Y, Kant R, Bairwa M. Determinants of COVID-19 vaccination willingness among health care workers: a quick online survey in India. Korean J Fam Med 2021;42:445-52.

7. Dror AA, Eisenbach N, Taiber S, Morozov NG, Mizrachi M, Zigron A, et al. Vaccine hesitancy: the next challenge in the fight against COVID-19. Eur J Epidemiol 2020;35:775-9.

8. Kwok KO, Li KK, Wei WI, Tang A, Wong SY, Lee SS. Editor's Choice: Influenza vaccine uptake, COVID-19 vaccination intention and vaccine hesitancy among nurses: a survey. Int J Nurs Stud 2021;114:103854.

9. Shekhar R, Sheikh AB, Upadhyay S, Singh M, Kottewar S, Mir H, et al. COVID-19 vaccine acceptance among health care workers in the United States. Vaccines (Basel) 2021;9:119.

10. Toth-Manikowski SM, Swirsky ES, Gandhi R, Piscitello G. COVID-19 vaccination hesitancy among health care workers, communication, and policy-making. Am J Infect Control 2021 Oct 13 [Epub]. https:// doi.org/10.1016/j.ajic.2021.10.004. 